\title{
Dynamic Road Traffic Signal Control using Digital Image Processing and RFID Sensors
}

\author{
Prof. Wrushali M Mendre ${ }^{1}$, Charudutta Sonone ${ }^{2}$, Shrikrishna Choudhari ${ }^{3}$, Kumar Gaurav ${ }^{4}$ \\ Smt. Kashibai Navale College of Engg, Pune, India ${ }^{1,2,3,4}$
}

\begin{abstract}
We all are experiencing heavy traffic in our metropolitan cities now a days. At such times it becomes very difficult for us to wait in heavy traffic jams. A solution to such conditions is given in our project, Dynamic traffic control using DIP n RFID sensors. At the traffic signals the count of the vehicles is taken (density count) and according to it, the duration of the traffic signal lights are modulated. Moreover for security purpose we are also using continues traffic monitoring and identification of the vehicle which has broken the signal is done using RFID sensors. Using GSM module and the data acquired from RFID module a penalty message is sent to the culprit with corresponding fine amount.
\end{abstract}

Keywords: Traffic density count, image processing, intelligent controlling of traffic, RFID module, GSM module.

\section{INTRODUCTION}

As seen in the above discussion the problem faced because of traffic jams is increasing day by day. Therefore it is very important to utilize the available resources with us very effectively. In metropolitan cities the problems faced because of traffic have increased considerably, and these problems can be crucial as well. For example an important person can be stuck in heavy traffic where he has to reach in time for an important meeting. Or consider an example of ambulance, where in if traffic jam is high, one cannot make way for the ambulance to pass. In such cases it can be a question of someone's life. For such situations an intelligent traffic control system is necessary. Even for the common public the time being wasted when we are stuck in traffic jam is not refundable. So finding solution and drawing a midway line through all the problems is must. A solution to such problems is discussed in the following paper. By intelligent traffic control, the paper suggests the idea that the duration of GREEN light or the RED light in our traffic signals can be modulated for heavy flows. Say for example on lane 1 there is heavy traffic and on lane 2 the traffic is comparatively low. In such conditions we can increase the time of GREEN light duration in lane 1 and we can reduce the time of same GREEN light in lane 2. wasting of the duration of GREEN light in lane 2 is inacceptable and inappropriate. Wastage of resources is being done. For avoidance of such wastage we are proposing our project where we modulate the duration of traffic lights for intelligent traffic flow. Various other papers have also discussed on the same topic with different techniques and those are discussed further. We in our paper are going to focus on density count of vehicles by using DIP and then modulating the Traffic Signals.

There are lots of techniques proposed to design an intelligent traffic system, for example, fuzzy based controller and morphological edge detection technique are proposed in [1] by Madhavi Arora, V. K. Banga, et. al. This technique is based on the measurement of the traffic density by correlating the live traffic image with a reference image. The higher the difference is, higher traffic density is detected. In [9] by Vikramditya Dangi, et. al. another technique is proposed to design an intelligent traffic system, which is based on four lane system in which time is allocated according to the number of vehicles on the lane. This paper also proposes an emergency vehicle detection, within a limited scenario. In another technique is proposed which is based on neural networks, which identify the vehicles and traffic density by processing the traffic videos. The technique proposed in is based on computing the traffic load by comparing two images, the reference image and the live traffic image. They improved object detection using image segmentation and noise removal operations.

In the discussed project, RFID module and GSM modules are also used for security purpose. If a vehicle has broken the Signal, there would be a RFID sensor near the ZEBRA crossing or some other deadline near the road. If the vehicle has crossed the deadline the RFID tag of that vehicle would be sensed and all the data of the owner of that vehicle would be obtained. Then using GSM module, a massage with prescribed fine amount would be sent to the responsible person and the fine would be acquired. Exception would be made for the emergency vehicles like ambulance or Fire extinguishers etc. If signal is broken by them then we would know by continuous traffic monitoring and also by their specific RFID tag.

The proposed paper is organized in the following pattern: First it focuses on the problems face in current scenario. Secondly it discusses about the technique used for its control. Thirdly it discusses about the methodology of the project and finally it talks about the results and conclusion made by the students and teacher Co-ordinator. 


\section{METHODOLOGY}

The project is divided mainly in 2 parts. The first part deals with the continuous video monitoring of the vehicles and calculating the density count of the road. After getting the density count the signal durations are modulated. And the second part deals with the violation of the traffic signal. If the signal is broken then the RFID tag of that concerned vehicle is sensed and through that information using GSM module a penalty message is being send. The basic working block diagram is shown below.

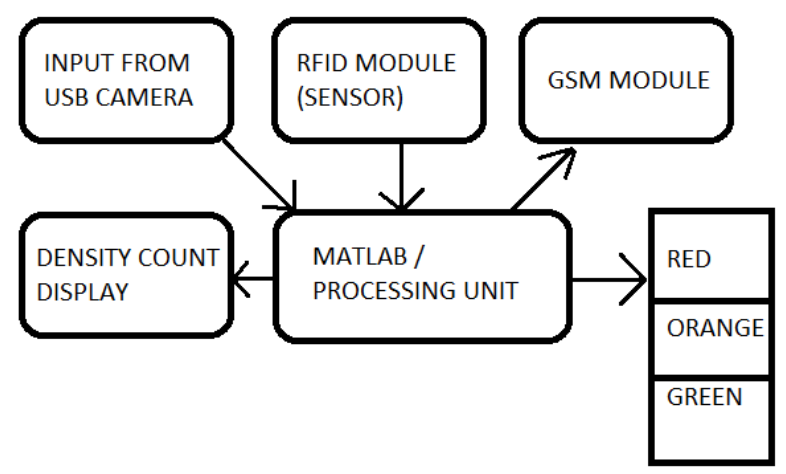

As shown in the block diagram Input from the USB camera is given to the processing unit. The processing unit or MATLAB after processing the snapshots calculates the density count and through a GUI displays the density count. Now according to the density count the duration of GREEN and RED lights is changed.

Further in part 2, If the RFID sensors detects a RFID tag that has broken the traffic signal it given affirmative signal to Processing unit and the processing unit initiates the GSM module to send the required complaint.

The processing work is divided into 4 parts. The first part is to process the continuous video signal and taking snapshot from fixed camera using MATLAB. In the second part by processing the acquired snapshot a threshold value of 500 is taken. The third part is the object detection which is performed by enhancing features of the image. Finally, the last part is the density counting, where the number of vehicles are being counted. The overall block diagram of the proposed system is illustrated below.

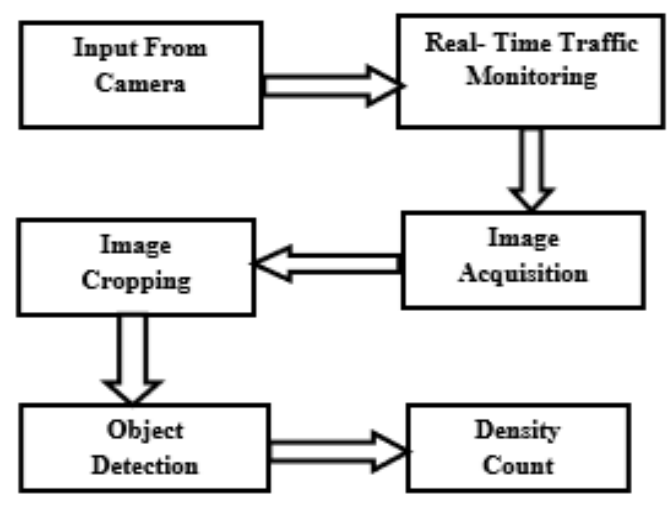

Fig 1: Block Diagram of the Proposed Model

\subsection{Processing of Video Signal and Image Acquisition}

In the very first stage the raw data extraction starts with the continuous monitoring of the road via video surveillance. This live video processing is done by using MATLAB software. The USB camera would be placed on the top of the traffic signal light or on the signal pole. The next part is to take images continuously from the video camera. This images are then further processed from RGB to GREY scale. Then a threshold value is calculated by comparing it with the earlier image of vacant road. Fig 2(a) shows the reference image which is captured from the live video when the road is empty. The next section explains the procedure to select region of interest where the vehicles are present.

\subsection{Image Cropping}

The second step is to select the targeted area by designing image cropping algorithms in MATLAB. The purpose of cropping is to identify the road region where the vehicles are present and exclude the unnecessary background information. This unnecessary information is fixed in every frame of the live video because the camera is stationary. To 
crop the required area, reference image has been used, Fig. 2(a), which has no road traffic. First, a binary image of having the same dimensions is created, as in the reference image, then the road area has been shaded white, and the leftover region as black, as shown in Fig. 2(b). Finally, the multiplication of the reference image with the cropping black and white image results in the final desired target area which is illustrated in Fig 2 (c).

The next part explains the procedure of object detection.

\subsection{Object Detection}

The third step is the object or vehicle detection in order to identify and count the vehicles which are present in the targeted area shown in Fig. 2(c). To perform the object detection, first the frame from the real time video sequence is extracted as illustrated in Fig 3(a). The next step is to convert both images; the reference image and the real time image into grayscale and then the absolute difference of two images will be determined. Since the dimensions of the road are fixed therefore the difference image only highlights the presence of vehicles in the desired target area. The difference image is illustrated in Fig. 3(b).

Fig. 3(b) shows the presence of vehicles in the desired target area but the visibility of the vehicles is not much clearer in that image. In order to improve the visibility of the vehicles, the difference image is converted to a binary image based on a threshold value. The resulting binary image is shown in Fig. 4(a), where the presence of any object is more improved. In order to determine only vehicles in the desired area, multiplication of the cropped image, Fig. 2(b), with the enhanced version of the difference image, Fig. 4(a), is carried out. The product image is illustrated in Fig. 4(b). In Fig. 4(b), the unnecessary information is filtered out and it only highlights the presence of vehicles in the desired area.

\subsection{Traffic Density}

The next step is to calculate the traffic density in the desired target area. In order to determine the traffic density, the vehicles are marked first and then their numbers are counted.

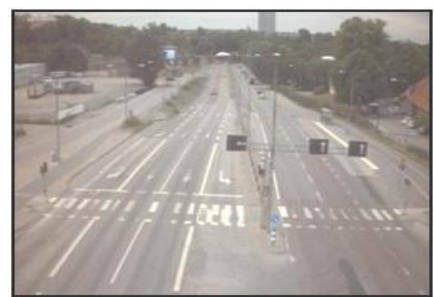

(a)

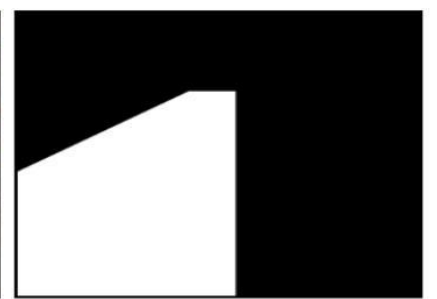

(b)

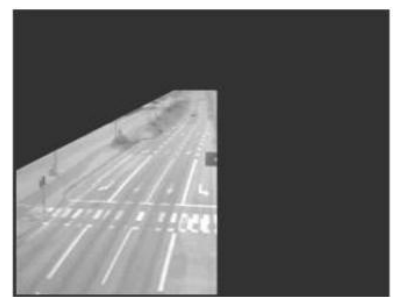

(c)

Fig 2: (a) Reference Image taken from the Live Video from [12], (b) Defining the region of interest, (c) Selection of the target area

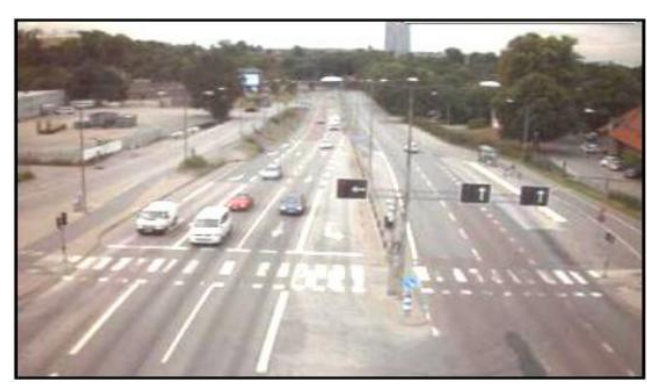

a)

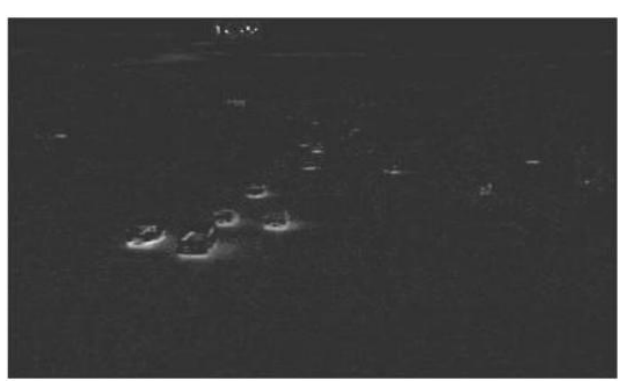

(b)

Fig 3: (a) Real-time image extracted from the live video

(b) Difference of reference and real time image

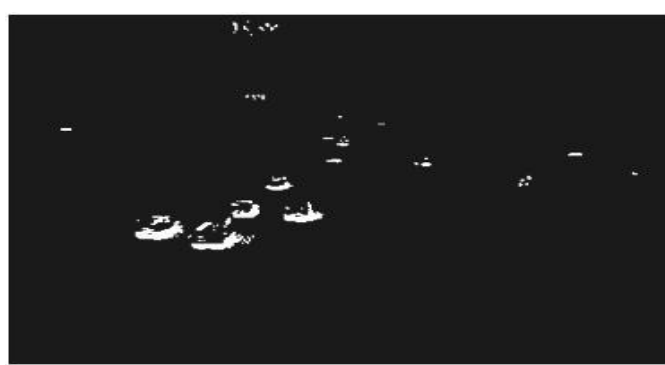

(a)

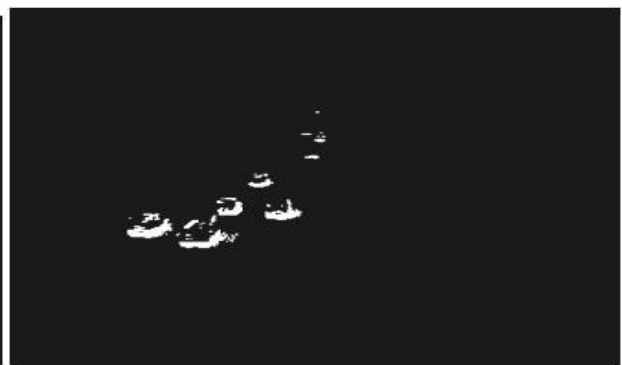

(b)

Fig 4: (a) Binarization of the difference Image, (b) Image highlighting the presence of vehicles in the targeted area 
The algorithm search for a set of connecting pixels. In order to consider a connected region as a vehicle, a minimum threshold has been defined. However, it is possible that more than one region of a vehicle is detected using the above criteria. This problem could be overcome by finding the overlapping bounding boxes of the selected regions and thus smaller and highly overlapping regions are filtered out. According to the density count the delay for the lights is programmed through MATLAB and traffic light duration changes. Some of the calculated results are shown in the RESULTS and DISCUSSION column. Further study is done later.

\section{RESULTS AND DISCUSSIONS}

The research is carried out in order to reduce the traffic congestion by calculating the traffic density in a particular direction of the road by using image processing algorithms. The system starts with an image acquisition process in which the live video is processed by the stationary camera, mounted on any pole. Then one frame per second continuously extracts from the live video and processed each frame by converting it into grayscale. For the reference image an empty road image was selected, when there is no traffic on the road. The second step is the image cropping in which, the targeted area is selected, the area where the vehicles are present and filtered out unnecessary surrounding information. Next phase, determines the presence of objects in live video by taking the absolute difference of each extracted frame with the reference image. Then the presence of objects is enhanced by binarization of the difference image. Then the final step is to calculate the traffic density in the desired target area by counting the number of vehicles in that region. To perform this, first, the vehicles are marked in the targeted region by scanning all the connected objects, and filtering out smaller and overlapping objects.

In order to deal with noise added due to different lighting conditions at different times of the day, a set of reference images have been captured and stored at different time slots of the day. The system cycles through these reference images according the current time of the day.

According to the current hardware implementation we have come up the following results for the project.

\begin{tabular}{|ll|}
\hline Density Count & Delay in Light \\
\hline 20 vehicles & $5 \mathrm{sec}$ \\
\hline 30 vehicles & $10 \mathrm{sec}$ \\
\hline 40 vehicles & $15 \mathrm{sec}$ \\
\hline 50 vehicles & $20 \mathrm{sec}$ \\
\hline
\end{tabular}

\section{CONCLUSION AND FUTURE WORK}

This paper discusses a method for estimating the traffic density on the lane by using image processing. The advantages of this proposed technique is that there is no need to use aerial imagery or complex sensor based systems. The proposed system is very cost effective as it does not require installation of any additional devices, such as RFIDs.

This work can be enhanced further by proposing a system which identifies the presence of emergency vehicles (like an ambulance or fire brigade) and by giving preference to those emergency vehicles. Secondly, it can be enhanced by using VANETs (Vehicular Ad-hoc Networks) as it provides road safety and intelligent transport system.

\section{REFERENCES}

[1] Madhavi Arora, V. K. Banga, "Real Time Traffic Light Control System", 2nd International Conference on Electrical, Electronics and Civil Engineering (ICEECE'2012), pp. 172-176, Singapore, April 28-29, 2012.

[2] Sabya sanchi kanojia, "Real -time Traffic light control and Congestion avoidance system", International Journal of Engineering Research and Applications (IJERA), pp.925-929, Vol. 2, Issue 2,Mar-Apr 2012.

[3] Muhammad Tayyab, "Implementation of Restoration Path Using AODV in VANETs" Master's Dissertation at Brunel University London, UK.

[4] Anthony J. Venables, "Evaluating Urban Transport Improvements", Journal of Transport Economics and Policy, Vol. 41, No.2, pp. 173-188, May, 2007.

[5] Tommy Gärling, Geertje Schuitema, "Travel Demand Management Targeting Reduced Private Car Use", Journal of Social Issues, Vol. 63, Issue 1, pp. 139-153, March 2007

[6] Papageorgiou M., Diakaki C., Dinopoulou V., Kotsialos, A.,"Review of road traffic control strategies”, Proceedings of IEEE, Vol. 91, Issue 12, pp. 2043-2067, November 2004.

[7] Georgios Vigos, Markos Papageorgioua, Yibing Wangb, "Real-time estimation of vehicle-count within signalized links", Journal of Transportation Research Part C: Emerging Technologies, Volume 16, Issue 1, pp.18-35, February 2008.

[8] Michael W. Szeto and Denos C. Gazis, "Application of Kalman Filtering to the Surveillance and Control of Traffic Systems", Journal of Transportation Science, vol. 6 pp.. 4419-439, November 1972.

[9] Vikramaditya Dangi, Amol Parab, Kshitij Pawar \& S.S Rathod, "Image Processing Based Intelligent Traffic Controller", Undergraduate Academic Research Journal (UARJ), Vol.1, Issue 1, 2012 